\title{
Los cuidados intensivos en el Antiguo Egipto a través de los textos*
}

\author{
Intensive care in Ancient Egypt through the texts \\ Cuidados intensivos no Antigo Egito através de textos
}

\begin{abstract}
Fernando Gil González ${ }^{1}$
${ }^{1}$ Licenciado en Historia. Colaborador Honorífico Departamento Historia Antigua. Universidad Complutense de Madrid. Cómo citar este artículo en edición digital: Gil González,F.(2012) Los cuidados intensivos en el Antiguo Egipto a través de los textos.16, 34. Disponible en: <http://dx.doi.org/10.7184/cuid.2012.34.08>

Correspondencia: Fernando Gil González.E-mail: fernando_gilgonzalez@hotmail.es

Recibido 09/05/2012/ Aceptado: 10/07/2012
\end{abstract}

\begin{abstract}
* Artículo correspondiente a la ponencia realizada en el III Simposio iberoamericano de historia de la enfermería, XII congreso nacional, VII internacional de historia de la enfermería y VII jornadas internacionales de cultura de los cuidados. Alicante 2011.
\end{abstract}

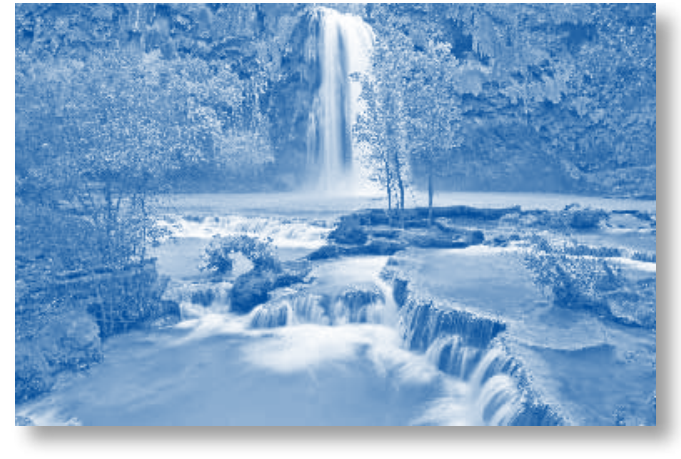

ABSTRACT

This article is entitled Intensive care in the Ancient Egypt through the texts. For this reason, they will try to elucidate various aspects of infectious treatments of the Pharaonic period through texts, conducting a comprehensive study on the treatments offered by the Egyptian doctors. To sum up, there will be a parallel between the Ancient Egypt's Medicine and the current one. Likewise you will defend a particular line of healing (instrumental, palliative, formulas), since ancient times until our days that will only be damaged by the use of the technological applications in the course of time.

Keywords: Medicine's History, Ancient Egypt, Intensive Cares, Texts.

\section{RESUMO}

Este artigo é intitulado Cuidados intensivos no antigo Egito através dos textos. Aí, eles vão tentar elucidar vários aspectos dos tratamentos infecciosos do período faraônico através de textos, conduzir um estudo abrangente sobre os tratamentos oferecidos pelos médicos egípcios. Em conclusão, haverá um paralelo entre a medicina do antigo Egito e o real. Da mesma forma, você vai defender uma linha particular de cura (fórmulas de paliativos, instrumental), desde a antiguidade até aos nossos dias, que só vão ser danificados pelo uso das aplicações tecnológicas no decorrer do tempo.

Palavras chave: História da Medicina; Antigo Egito; Cuidados Intensivos, Testamentos.

\section{RESUMEN}

El presente artículo lleva por título Los Cuidados Intensivos en el Antiguo Egipto a través de los textos. En él, se intentarán dilucidar diversos aspectos de los tratamientos infecciosos en el periodo faraónico a través de los textos, realizando un exhaustivo estudio sobre los tratamientos ofrecidos por los médicos egipcios. Como conclusión, se realizará un paralelismo entre la Medicina del antiguo Egipto y la Ac- 
tual. Así mismo se defenderá una determinada línea de curación (instrumental, paliativos, fórmulas) desde la antigüedad hasta nuestros días, que sólo se verá afectada por el uso de las aplicaciones tecnológicas en el transcurso de los tiempos.

Palabras clave: Historia de la Medicina, Antiguo Egipto, Cuidados Intensivos, Textos.

"(Thot) hace que hablen los escritos, crea la colección de recetas $(y)$ da el poder a los sabios (y) a los médicos que están a su servicio para liberar (del mal) a aquel a quien dios ama (Ebers; 1 ; 8-10)".

\section{INTRODUCCIÓN}

En el presente artículo se pretende realizar un estudio sobre los cuidados intensivos en el antiguo Egipto, a través de una visión histórica, donde se describen los procedimientos médicos para combatir las enfermedades en la antigüedad.

Con la ayuda de los textos, principalmente con el empleo de los Papiros Médicos, se interpretarán de una forma objetiva los tratamientos de las enfermedades desarrolladas en el Egipto faraónico. La tipología textual es abundante y en ella se pueden resaltar elementos solventes (Papiro Ebers, Papiro Londres, Papiro Westcar, Papiro Smith, etc.) con los que se obtendrán distintas informaciones, según las cuales se construirá el presente artículo. El método a emplear es significativo. Está basado en el uso de las distintas fuentes textuales, con el fin de ahondar en las diversas interpretaciones que ofrecen los Papiros médicos del antiguo Egipto. También se usan fuentes primarias, como textos de Heródoto, Diodoro de Sicilia, Estrabón, El Antiguo Testamento etc., que ofrecen ciertos datos relevantes sobre la
Medicina en el antiguo Egipto (Leca, 1986) Como colofón, se intenta comparar el sistema médico egipcio con el actual, basándonos en la existencia de distintos tratados médico-quirúrgicos que versan sobre los diferentes elementos anatómicos del cuerpo humano, apreciados en los Papiros Médicos (Da Silva, 2009)

Los objetivos de este trabajo se centran en el propósito de analizar el sistema de curación del antiguo Egipto y compararlo con el sistema médico actual, mediante el empleo de las fuentes clásicas literarias y el análisis de los papiros médicos traducidos por los especialistas, que detallan los sistemas curativos de la antigüedad. Mediante la ayuda de distintas aportaciones históricas, literarias, arqueológicas, médicas etc..., se pretende realizar un estudio interpretativo determinando que la ciencia médica del antiguo Egipto es similar a la que se realiza en los tiempos actuales. Esta idea es plausible debido a que el instrumental, sistemas de curación, fórmulas, prescripciones etc... son muy similares a los empleados en la Medicina actual. Finalmente se determinará que el uso y empleo de los medios curativos desde la antigüedad se ha ido desarrollando en distintas líneas tras los continuos avances tecnológicos, con el paso del tiempo, que permiten la comprensión de un oscuro tema como es la Medicina egipcia.

\section{ESTADO DE LA CUESTIÓN/ANTECE- DENTES DEL TEMA}

La salud y las prácticas médicas en el antiguo Egipto están inmersas en diversos aspectos entre los que destacan la Religión, la Arqueología o los autores clásicos (Tito Flavio Clemens, 1, XVI). En la antigüedad, la Medicina egipcia tiene un origen complejo. Proviene de las prácticas sanitarias de las colonias fenicias o sirias, que han sido copiadas en las tierras del 
Nilo (Kemet). En los libros/manuscritos/papiros se puede observar una serie de diagnósticos y terapias que se han ido reconstruyendo a lo largo de los siglos. Clemente de Alejandría, describe la constitución del cuerpo humano, patologías, tipos de órganos, prescripciones, tratamientos etc... Aparte de la recopilación textual, se pueden encontrar elementos iconográficos como tradiciones, mitos, cosmogonías localizados en el periodo predinástico. Desde el siglo IV d. C. en la ciudad de Alejandría se pueden realizar autopsias y disecciones corpóreas como se reflejan en texto localizados en su Biblioteca pertenecientes a autores destacados como Areteo de Capadocia, Heródoto, Diodoro de Sicilia etc... (Da Silva, 2009) Durante el periodo contemporáneo, se localizan mejor las enfermedades tras el estudio de los distintos papiros/manuscritos encontrados. J.F. Champollion en su expedición a Egipto encuentra la Piedra Rosetta, que permitió traducir los jeroglíficos a las lenguas modernas. Además describe monumentos y halla una serie de papiros con distintas enfermedades corpóreas en las que se aprecia una fuerte influencia espiritual (Denon, 2004).

Es importante resaltar la obra de Wilkinson que en 1847 determina la existencia de unas pocas enfermedades en el antiguo Egipto. Las más destacadas son disentería y las pandemias referentes a la Oftalmología. También su libro presenta prescripciones médicas y la protección de dichas enfermedades. Desgenettes explica en sus escritos la existencia de una estricta higiene en las prácticas médicas, el uso de profilácticos, limpieza en la ropa y control de la higiene alimentaria (Da Silva, 2009). También se puede apreciar en sus textos las distintas enfermedades como plagas, disentería, conjuntivitis etc... A. Barthelemy Clot localiza en los papiros el empleo de la disecci- ón de cadáveres (parecido a las trepanaciones egipcias), distintas prescripciones médicas, ingredientes, recitales mágicos y fórmulas rituales. Por último, en el siglo XX, F. Jonkheere describe las enfermedades egipcias y las prácticas del antiguo Egipto mediante el empleo de la higiene y los avances biomédicos (Da Silva, 2009). Como conclusión, se debe determinar que el sistema de curación no dista demasiado del sistema médico actual determinando que la curación es bastante similar basándose en las pruebas expuestas.

\section{MÉTODO}

Se trata de un estudio de carácter histórico en el que se puede apreciar un análisis de los documentos presentados. Como fuentes primarias se han empleado los papiros médicos, los tratados clásicos de Heródoto, Homero etc... con el fin de ofrecer al lector los testimonios de la época. Finalmente como fuentes secundarias se emplearán los escritos de los autores modernos y destacados especialistas, que han examinado los documentos antiguos, interpretándolos de distinta forma. Tras el análisis documental se debe concluir que la Medicina egipcia no es tan antigua per se, sino que forma parte de una misma línea de curación instaurada desde las culturas mesopotámicas hasta el periodo actual.

\section{RESULTADOS Y DISCUSIÓN}

El médico o sanador (Swnw o Sunu) es un personaje de gran relevancia en el periodo faraónico (Calvo, 2003). Se trata de un funcionario con conocimientos técnicos que corrige una cura (Sánchez, 2001). Éstos se clasifican por categorías dependiendo de su rango (Luca, 1986) En los casos de tratamientos de procesos infecciosos intervienen también los Sacerdotes de Sejemet, que son veterinarios o médicos 
(Luca, 1986). Entre los más conocidos podemos citar a Hesire (Sánchez, 2001) Los sanadores realizan sus tratamientos en las Per-Ankh o Casas de la Vida (Da Silva, 2009), que se localizan en determinados puntos del "Doble País" (Heliópolis y Sais).

Heródoto, en sus escritos, refiere la existencia de algunos tipos de especialidades médicas: Unos son de los ojos, otros de la cabeza, otros de los dientes; otros de las enfermedades abdominales etc.... (Historia; II; 84)

También se ocupaban de los tratamientos de embalsamiento y momificación de los cadáveres (Da Silva, 2009) Homero relata en La Odisea: Los médicos egipcios eran más hábiles que los de otras tierras. En el Papiro Ebers se puede contemplar otra inscripción similar (Bardinet, 1995) Desde Iunu (Heliópolis) salí con los Grandes de los templos-señores de la protección (y) gobernadores de la eternidad. Es cierto, salí (también) desde Sais con las madres de los dioses. Me dieron sus conocimientos. (Ahora) me pertenecen los preceptos que dictaminó el señor del Universo para eliminar (las enfermedades) (Ebers, 1, 1-3) Plinio el Joven, en su Historia Natural, refiere la existencia de fármacos (sustancias) y de procedimientos terapéuticos (Luca, 1986).

Para concluir el instrumental empleado en el antiguo Egipto es muy significativo (bajorrelieves del templo de Kom Ombo). La presencia

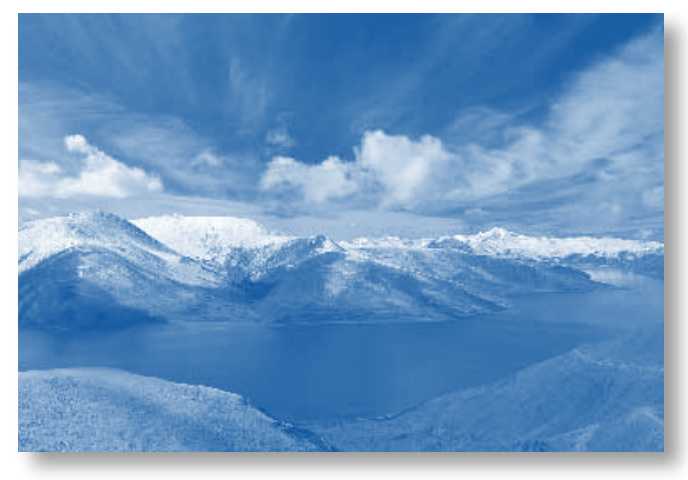

de sierras, bisturíes, tijeras etc... realizados en bronce y obsidiana datan de una antigüedad superior a cuatro mil años (Ruiz et alii, 2008) Éstos pretenden compararse a los que se construyen en la actualidad de acero quirúrgico, determinando que su actuación, precisión y funcionalidad es bastante similar tras los análisis médico-quirúrgicos realizados en las momias (Nunn, 1996)

\section{LA ENFERMEDAD EN EL ANTIGUO EGIPTO}

Las enfermedades pueden cursar a través de la obstrucción de los conductos sanguíneos provocando así el envejecimiento de la persona. En el caso femenino también se pueden obstruir los conductos sexuales, produciéndose la infertilidad. El peor síntoma para los egipcios es el estreñimiento y sólo se puede evitar con la purga. Para ello, los médicos egipcios estudiaban las heces de los enfermos y explicaban sus síntomas. La enfermedad también puede aparecer por la acción de diversas divinidades malignas, provocando daños en los fluidos corporales. Otras causas para contraer la enfermedad son: el castigo divino, el exceso de tristeza, el mal de amores, el mal de ojo, etc. Finalmente, las enfermedades causadas por la naturaleza son muy frecuentes, como el hambre, la ingesta de alimentos en mal estado o el sobreconsumo de cerveza. (Cuenca-Estrella et alii, 2004)

Existen más de doscientas clases de enfermedades en el antiguo Egipto. Las más conocidas son las heridas y las fracturas. Las dolencias por síntomas no se consideran debido a que, hasta mediados del siglo XIX no se utilizó este sistema de clasificación técnica curativa.

El Papiro Smith contiene un Libro de las Heridas, en donde se estudian cuarenta y ocho casos de lesiones traumáticas (Cuenca-Estrella 
et alii, 2004) Posee también un Tratado de Cirugía y Traumatología en donde se estudian traumatismos craneales y algunas operaciones quirúrgicas. En el Papiro Ebers encontramos descripciones de padecimientos digestivos, estreñimientos y textos referidos a enfermedades del Aparato Genitourinario y Respiratorio (Bardinet, 1995) Las enfermedades cardiovasculares, son también conocidas en el antiguo Egipto. Las más citadas son los edemas en las extremidades inferiores, la insuficiencia cardiaca o los infartos agudos de miocardio. En cuanto a las enfermedades neurológicas, el $\mathrm{Pa}$ piro Ebers trata diversas cefaleas, demencias, convulsiones, temblores y parálisis faciales.

\section{ENFERMEDADES EN EL ANTIGUO EGIPTO}

Para el estudio de las enfermedades, se plantean tres vías de investigación:

1) El estudio radiológico de las momias y de sus tejidos para conocer el motivo de la enfermedad o muerte del paciente.

2) Representaciones de malformaciones (Reina Hapshepsut con Esteatopigia severa).

3) Papiros Médicos (Ebers, Smith, Kahún...)

Las dolencias más frecuentes del antiguo Egipto son las siguientes: Esquisomiasis, Helmintos, Tuberculosis, Poliomielitis, Artritis, Ceguera y Enanismo. La Ceguera es contemplada en el oficio del músico, como el Arpista ciego de la tumba de Najt (Sánchez, 2001) Las enfermedades degenerativas son bastante conocidas como la Silicosis, la Arteriosclerosis, cuadros de cefaleas, migrañas o ataques epilépticos (Da Silva, 2009).

\section{LOS TRATADOS MÉDICOS: LOS PAPIROS}

Los Papiros Médicos son guías para conocer y tratar las distintas enfermedades existen- tes en el Antiguo Egipto.

El Papiro Ebers (1550 a.C.) es un compendio farmacológico y fisiológico (Bardinet, 1995) Este papiro formado por hojas de un tamaño de $20 \times 23 \times 30 \mathrm{~cm}$. Se trata de un papiro copiado en el noveno año de Amenhotep I. En 1872 pasa a ser propiedad de G. Ebers siendo un libro extenso y deslavazado. A pesar de ello, describe importantes enfermedades y el tratamiento de las mismas. Las recetas más importantes que aparecen, son de ámbito mágico-religioso y se elaboran con ciertos elementos animales, vegetales y minerales. Además de su composición, se indica la preparación y la forma de ingestión del preparado. Además este papiro trata enfermedades estomacales (Ebers, 36, 4-6).

El Papiro Hearst, es un documento que contiene unas doscientas sesenta inscripciones, en las que contemplan distintas recetas.

El Papiro Médico de Berlín (3038) pertenece a la dinastía XIX, y de él, sólo se conservan unas doscientas cuatro recetas.

El Papiro de Kahún (erróneamente llamado) es del 1850 a. C., durante el $29^{\circ}$ año de reinado de Amenenhat (Cuenca-Estrella et alii, 2004). En él se localizan treinta y cuatro recetas.

El Papiro Médico de Londres o BM 10.059, es coetáneo a la época de Tutankhamón y en él, se aprecian diversos procedimientos mágicos para paliar las enfermedades. El Papiro Chester-Beatty, data del 1300 a. C, dónde se contemplan cuarenta y cinco preinscripciones y encantamientos contra la migraña.

En los Papiros III y IV del Rameseum, se localizan enfermedades ginecológicas. Por último, el Papiro Smith que data del año 1500 a.C. Es un manuscrito de unas diecisiete páginas y en él, se aprecian distintos tratamientos quirúrgicos. Es un tratado de la época dinásti- 
ca dónde se presentan diversos tipos de curas: heridas, pequeñas operaciones, contusiones, entablillado de fracturas y eliminación de pequeños tumores. En definitiva es una guía médica muy completa (cuarenta y siete diagnósticos, ocho encantamientos, fabricación de cosméticos y una preinscripción de patología anal) (Sánchez, 2001)

\section{REMEDIOS Y ACTUACIÓN MÉDICA EN EL ANTIGUO EGIPTO}

Los remedios para las actuaciones médico-quirúrgicas son limitados (Fernández, 1999) No sólo hay recetas mágicas y religiosas, sino que se prescriben ciertos preparados naturales o Sheausau (Nunn, 1996) En la actuación médica, el sanador explora al paciente (inspección de manchas, signos de enfermedad, existencia de traumatismos...) Posteriormente reconoce el aliento del paciente y le obliga a realizar diversos ejercicios físicos. Finalmente le escuchaban el latido del corazón y los movimientos respiratorios. La consulta médica podría realizarse en el domicilio del enfermo aunque dicho dato lo desconocemos por completo (Fernández, 1999) En los textos como el Papiro Ebers, se aprecian ochocientas setenta y siete sustancias diferentes para efectuar la cura (prescripciones, remedios, tratamientos, consejos médicos, etc....) Si no mejora el enfermo, se le aplica un tratamiento psicológico. El sanador realiza posteriormente una Rogativa (Cuenca-Estrella et alii, 2004) Finalmente, si todo falla, es necesario recurrir a la Cirugía. Tras esto, el médico aplica un método quirúrgico distinto para cada día del año, basándose en el calendario egipcio, concluyendo con una operación quirúrgica aunque ésta a veces estaba conectada directamente con la muerte del paciente (Kolta, 2000)

Por último, se indica la existencia de unos medicamentos arcaicos (Nunn, 1996) en- tremezclados con hechizos o encantamientos para paliar las enfermedades (Cuenca-Estrella et alii, 2004) Estos preparados eran distribuidos en distintas cantidades (Heqat, Henu, Ro etc.) (Nunn, 1996) con el objeto de controlar las dosis de los pacientes como ocurre de forma análoga en la actualidad, y en diferentes días (fastos e infaustos) según su efecto.

El sistema médico actual ofrece una serie de cuidados prescriptos por el médico quien receta a sus pacientes distintos fármacos con el fin de paliar su enfermedad.

\section{CONCLUSIONES}

El médico responsable emplea unos procedimientos médico-mágico-religiosos para paliar las enfermedades de los pacientes, ayudado por sus adjuntos enfermeros y veterinarios (sacerdotes).

Se defiende la existencia de un sistema médico-quirúrgico bastante complejo, gracias al instrumental utilizado y las técnicas curativas así como los protocolos de curación empleados en el antiguo Egipto, muy similares a los actuales.

Se considera que los médicos egipcios usaban una documentación similar a la de una especie de vademécums o manuales de Medicina, en donde se describen las enfermedades más frecuentes en el antiguo Egipto.

A veces las pandemias no se podían determinar, localizar ni solventar por los elementos naturales, sino que era necesario emplear algunos remedios de índole mágico-religiosa, con el fin de salvar al paciente. En las situaciones más graves el médico solía citar la frase No estoy capacitado para corregir la enfermedad, provocando la muerte del paciente.

Parece plausible que el sistema médico egipcio, sea un sistema de curación por órga- 
nos y, por esta razón, no se trata de un sistema universal.

Determinar la inexistencia de medicamentos, sensu strictus, en el antiguo Egipto. Dichos elementos curativos, son paliativos y recetas artesanales con distintos compuestos (vegetales, animales y minerales). Se usan como distintos preparados y paliativos que ayudan a sanar al paciente, tras un efecto psicológico, mediante el uso de diversas fórmulas rituales y mágicas (algunos de ellos, por supuesto, no son más que placebos que influyen en la predisposición psíquica del paciente para favorecer su curación) Es importante citar el estrecho contacto entre la Medicina y la Religión, por las presentes conexiones rituales en los sistemas de curación de los antiguos egipcios.

Cómo conclusión final se debe determinar que existe una misma línea de curación entre el antiguo Egipto y el periodo actual, en lo que respecta a la forma de realizar las curas, aplicación de distintos ungüentos y paliativos, recital de fórmulas, prescripciones etc...El único distintivo que puede existir es la aplicación tecnológica-según su contexto histórico-en los distintos preparados o en la construcción de los instrumentos que instan a realizar la curación médica.

\section{BIBLIOGRAFÍA}

- Bardinet, T. (1995) Les Papyres Médicaux de 1' Egypte pharaonique. Libraire Arthéme Fayard.

- Calvo Soriano, G. (2003) La medicina en el Antiguo Egipto; PAEDIATRICA

- Asociación de Médicos Residentes del Instituto Especializado de Salud del Niño, 44-50.

- Cuenca-Estrella, M. y Barba, R. (2004) La Medicina en el Antiguo Egipto. Alderabán. Colección: El Legado de la Historia (43), Madrid.

- Da Silva Veiga, P.A. (2009) Health and Medicine in Ancient Egypt. Magic and science. BAR International Series 1967.
- Denon, D.V. (2004) Viagem au Baixo e Alto Egyt. Publicações Europa-America, Lisboa.

- Fernández Uriel, P. (1999) (Coord. Ángeles Alonso Ávila) Males y remedios: Notas sobre la medicina en el mundo antiguo: Próximo Oriente y Egipto. Homenaje al profesor. Á. Montenegro: Estudios de Historia Antigua, 17-30.

- Heródoto (1992) Historia: Libros I-IX; Gredos, Madrid.

- Homero (1992) La Odise. Gredos, Madrid.

- Kolta, K. S. (2000) Die Heilkunde im Altem Ägyten; Steiner, Alemania.

- Leca, A. P. (1986) La Medizina Egizia. Al tempo dei Faraoni. Ciba-Geigy Edizioni, París.

- Nunn, F. (1996) Medicina del Antiguo Egipto. Sección de obras de ciencia y tecnología. Fondo de Cultura Económica, Londres.

- Ruiz Bremón, M. y San Nicolás Pedraz, Ma P. (2008) Enfermar en la Antigüedad. Universidad Nacional de Educación a Distancia, Madrid.

- Sánchez Rodríguez, Á. (2001) La Medicina en el A. Egipto. Terralia, 21, 67-73. 